\title{
SKETCH BASED IMAGE RETREIVAL SYSTEMS : A COMPARATIVE STUDY
}

\author{
Safiya P.C \\ MTech Computer Science, KMCT College of Engineering, Calicut, Kerala, India.
}

\begin{abstract}
Image retrieval systems which uses image sketches as the input are known as sketch based image retrieval (SBIR) systems. Main advantage of SBIR system is that it does not need to have a high skill to draw a query sketch. One of the main challenges in sketch-based image retrieval (SBIR) is to compute the similarity between a query sketch and a database image. To deal with this problem, we propose a Sketch Based Image Retrieval approach by salient contour reinforcement. In the proposed method, the image contour is divided into two types namely global contour map (GCM) and the salient contour map (SCM). SCM is helpful to find out the object inside images which are similar to the query image. In addition to two types of contour maps we proposes a new feature called angular radial orientation partitioning (AROP) feature. AROP feature fully utilizes the edge pixel's orientation information in GCM and SCM to identify the spatial relationships. Our AROP feature based on GCM and SCM is very effective to discover false matches of local features between query sketch and database images, and have a huge improvement in the retrieval performance. This paper presents a review and a comparison of few of the various SBIR methods commonly used in image processing. SBIR systems are very effective in the field of medical diagnosis, photos haring sites, digital library etc.
\end{abstract}

Keywords: SBIR, Salient Contour, Contour Reinforcement

\section{INTRODUCTION}

Digital Image processing is the manipulation of images from a digital camera. Image retrieval means retrieving images from digital image database by searching for desired images. In order to search for an image, we need to provide an image or an image description as a search query. The image retrieval system will return images "similar" to the query after searching in the digital database. The similarity can be measured based on meta data, the contents or the shape of an image.

With the advances in networking and multimedia technologies, the demand for effective and efficient image retrieval systems or tools also have increased. In order to retrieve the images we need from large digital image database we use Content Based Image Retrieval (CBIR) systems or tools. The state of the art CBIR systems use an image as a query. CBIR indexes the images based on the features obtained from visual content so as to facilitate speedy retrieval. When the user is not able to provide the exact image which he wants to query for or when he lacks the ability to describe the image as expected, it is difficult to search and retrieve the intended image from digital image database. To tackle this problem, we propose a new image retrieval system based on query sketch. That is the user needs to simply draw a sketch of the intended image and give the sketch as the query. The system returns the similar images by matching the shape or sketch of the images.

SBIR systems can be used to take a hand drawn sketch as the input query and retrieve the similar images from large scale digital database. Human brain recognizes an object based on its shape or contour. We can draw the shape of an object in simple strokes for any of the natural scene images. But there may exists some ambiguities in sketches due to poor drawing skills. To remove these ambiguities the image retrieval system should be able to deal with the ambiguity existing if any.

Traditional draw-and sketch systems need to have a query sketch which is similar to natural scene image to be searched. That is the sketch should be similar in color shape and texture. This converts the problem of sketch based image retrieval to content based image retrieval (CBIR). The major goal of SBIR technology is to measure the similarity between a hand drawn sketch and an image. That is by measuring the contour similarity between input query sketch and the database image. Thus the problem of SBIR is converted to contour matching. Shape of an object have an important role in image comparison and is used in object detection and comparison. Shape of an object can be described using shape descriptors or shape contexts. Shape descriptors can be global or local. Global descriptors plays a major role in image analysis.

In the proposed SBIR system, the main focus in on search precision. To retrieve more precise images, image representation and image matching has to be done efficiently. Image contours and orientation are the two major concepts which is exploited in SBIR technology. Structure of an image along with its texture can be studied using image contours. Orientation has been widely exploited due to its outperforming results in object detection and categorization. The method, the image contour is divided into two types namely global contour map (GCM) and the 
salient contour map (SCM). SCM is helpful to find out the object inside images which are similar to the query image.In addition to two types of contour maps we proposes a new feature called angular radial orientation partitioning (AROP) feature. AROP feature fully utilizes the edge pixel's orientation information in GCM and SCM to identify the spatial relationships. Our AROP feature based on GCM and SCM is very effective to discover false matches of local features between query sketch and database images, and have a huge improvement in the retrieval performance.

In this paper I conducted comparative study on different Image retrieval systems. The main contributions of this paper is a review of few of the SBIR systems namely 1 . Query by Visual Example (QVE), 2. SBIR approach with re-ranking and relevance feedback schemes, 3 . Composing realistic pictures from free hand sketch and 4. Edgel index approach.

\section{SKETCH BASED IMAGE RETREIVAL (SBIR) SYSTEMS}

A sketch is a free hand-drawing consisting of a set of strokes. A sketch lacks color and texture. Sketch has been drawn without filling or shadows. Drawing a query sketch is an easy task in today's electronic era.. The retrieval system using sketches can be effective and essential in our daily life in various fields such as Medical diagnosis, photo sharing sites, digital library, search engines, geographical information, and sensing remote systems. Here I have done a comparative study about QVE, Re-ranking and relevance feedback, composing realistic picture from free hand sketch and edgel index method.

\subsection{Qve}

Query by Visual Example is one of the early approaches in SBIR. This method is used when the image can be represented as a bag of words. QVE method uses blocks and local features for image comparison and retrieval. The process is accomplished in four steps. 1. Re-size the query image and database images into $64 \times 64$ pixels. 2. Extract the edges using gradient operator 3 . correlation is calculated by shifting these blocks 4. Edge histogram descriptor and histogram of oriented gradients are used. Other local descriptors can also be defined to achieve high precision in image retrieval.

\subsection{SBIR With Re-Ranking And Relevance}

\section{Feedback}

The machine learning algorithm is used to take the feedback from user. If the query image is a system as well as the text, then the users can check the relation between image and the query. Method employs a binary classifier which labels the images as relevant or irrelevant. Then a dimension reduction is conducted which localizes the visual features of the labeled image. Multiple iteration of the above processes increases the accuracy of the retrieved results.

\subsection{Realistic Picture From Free Hand Sketch}

There is another image retrieval system which composes realistic pictures from a simple free hand sketch annotated with text labels. In this system the retrieval process is done as follows. 1. Firstly, relevant images and backgrounds are searched using textual keywords. 2. from the search results, candidate images are chosen. 3 . Then during filtering, each image is segmented to find scenic image corresponding to the sketch. 4.Then the combination of filtered images is optimized into a picture by two steps. a) Optimize the blending boundary and assign each pixel within the boundary a set indicating whether the texture and color of the pixel are consistent. b) compute the blending result by combining improved position blending and alpha blending.

\subsection{Edgel Index Approach}

Edgel Index approach is a shape based approach in which the image retrieval is done by comparing the shape of images. Pixel level matching is done for solving the shape to image matching problem. Edgel is the short form for edge pixel. Each visual word is described using the triple $(\mathrm{x}, \mathrm{y}, \theta)$ of the position $(\mathrm{x}, \mathrm{y})$ of an edgel and the edgel orientation $\theta$ at that position.

\section{COMPARISON OF VARIOUS SKETCH BASED IMAGERETRIEVAL METHODS}

In this review paper few of the image retrieval methods based on sketch of the query image is discussed and a comparison of thesemethods are given in TABLEI.

Table -1: Different methods for SBIR

\begin{tabular}{|c|c|c|c|}
\hline Method & $\begin{array}{l}\text { Feature/ } \\
\text { Methodolo } \\
\text { gy }\end{array}$ & Advantages & $\begin{array}{l}\text { Disadvantag } \\
\text { es }\end{array}$ \\
\hline QVE & $\begin{array}{l}\text { Local } \\
\text { features for } \\
\text { image } \\
\text { compariso } \\
\mathrm{n}, \quad \text { Edge } \\
\text { histogram } \\
\text { descriptor } \\
\text { and } \\
\text { histogram } \\
\text { of oriented } \\
\text { gradients } \\
\text { are used. }\end{array}$ & $\begin{array}{l}\text { No need of } \\
\text { providing } \\
\text { effective } \\
\text { descriptions. }\end{array}$ & $\begin{array}{l}\text { images that } \\
\text { share } \\
\text { similar sets } \\
\text { of regions } \\
\text { are deemed } \\
\text { to be related } \\
\text { even when } \\
\text { the depicted } \\
\text { actions are } \\
\text { different }\end{array}$ \\
\hline $\begin{array}{l}\text { Re- } \\
\text { ranking } \\
\text { and } \\
\text { Relevan } \\
\text { ce } \\
\text { Feedbac } \\
\text { k }\end{array}$ & $\begin{array}{l}\text { Machine } \\
\text { learning } \\
\text { algorithm } \\
\text { is used, As } \\
\text { per the } \\
\text { feed back } \\
\text { from the } \\
\text { user, the } \\
\text { images are } \\
\text { displayed } \\
\text { in order }\end{array}$ & $\begin{array}{l}\text { Collect labeling } \\
\text { information from } \\
\text { user toobtain } \\
\text { specified } \\
\text { semanticspace. } \\
\text { Localize the } \\
\text { visualcharacterist } \\
\text { ics of the user } \\
\text { intentions in } \\
\text { space. }\end{array}$ & $\begin{array}{l}\text { Need user } \\
\text { to provide } \\
\text { feed back } \\
\text { for effective } \\
\text { re-ranking }\end{array}$ \\
\hline
\end{tabular}




\begin{tabular}{|c|c|c|c|}
\hline $\begin{array}{l}\text { Realistic } \\
\text { pictures } \\
\text { from } \\
\text { free } \\
\text { hand } \\
\text { sketch }\end{array}$ & $\begin{array}{l}\text { Compose } \\
\text { realistic } \\
\text { pictures } \\
\text { from free } \\
\text { hand } \\
\text { sketch } \\
\text { using } \\
\text { textual } \\
\text { search }\end{array}$ & $\begin{array}{l}\text { User can use any } \\
\text { object label for } \\
\text { image retrieval, } \\
\text { allows finer } \\
\text { control } \\
\text { over the objects' } \\
\text { appearance. }\end{array}$ & $\begin{array}{l}\text { Obtaining } \\
\text { human } \\
\text { annotations } \\
\text { is expensive } \\
\text { and time } \\
\text { consuming }\end{array}$ \\
\hline $\begin{array}{l}\text { Edgel } \\
\text { Index } \\
\text { Approac } \\
\text { h }\end{array}$ & $\begin{array}{l}\text { Shape } \\
\text { based } \\
\text { approach, } \\
\text { solves } \\
\text { shape to } \\
\text { image } \\
\text { matching } \\
\text { problem } \\
\text { using pixel } \\
\text { level } \\
\text { matching. }\end{array}$ & $\begin{array}{lr}\text { Builds } & \text { an } \\
\text { efficient } & \text { index } \\
\text { structure } & \text { for } \\
\text { large } & \text { scale } \\
\text { retrieval } & \end{array}$ & $\begin{array}{l}\text { Doesn't use } \\
\text { affine } \\
\text { invariant } \\
\text { shape-to- } \\
\text { image } \\
\text { matching }\end{array}$ \\
\hline
\end{tabular}

\section{CONCLUSIONS}

As a review paper, this work mainly focused on different methods or approaches used in SBIR technology. Each method has its own advantages and disadvantages as it may be for a specific purpose. Main methods along with the limitations are analyzed here and since each one has its own disadvantages I propose a new method to implement SBIR in which image retrievals done by salient contour reinforcement.

\section{ACKNOWLEDGEMENT}

The author would like to acknowledge Mr. Niyas $\mathrm{N}$ and $\mathrm{Mr}$. Swaradh P of Department of computer science and Engineering, KMCT College of Engineering Calicut, for their valuable help and suggestions in conducting this study.

\section{REFERENCES}

[1]. X. Qian, Y. Zhang, Xianglong Tan, Junwei Han, and Yuanyan Tang "Sketch-based Image Retrieval by Salient Contour Reinforcement" IEEE TRANSACTIONS ON MULTIMEDIA, MM-006447 .

[2]. K. Hirat, and T. Kato, "Query by visual example," Advances in Database Technology, EDBT'92. Springer Berlin Heidelberg, 1992, pp. 56-71.

[3]. X. Qian, X. Tan, Y. Zhang, R. Hong, and M. Wang, "Enhancing Sketch-Based Image Retrieval by Reranking and Relevance Feedback", IEEE Trans. Image Processing, 2015. pp.195-208.

[4]. Y. Cao, C. Wang, L. Zhang, and L. Zhang, "edgel index for large-scale sketch-based image search," IEEE CVPR, 2011, pp. 761-768.

[5]. T. Chen, M. Cheng, P. Tan, A. Shamir, and S. Hu, "Sketch2Photo: internet image montage," ACM Transactions on Graphics (TOG), 2009, pp.89-97.

[6]. Mayuri D. Joshi, Revati M. Deshmukh, Kalashree N.Hemke, Ashwini Bhake and Rakhi Wajgi, "IMAGE RETRIEVAL AND RE-RANKING TECHNIQUES -
A SURVEY", Signal \& Image Processing : An International Journal (SIPIJ) Vol.5, No.2, April 2014.

[7]. David Engel, Christian Herdtweck, Björn Browatzki and Cristóbal Curio, "Image Retrieval with Semantic Sketches",

\section{BIOGRAPHIES}

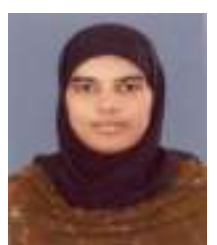

Safiya PC received the B.Tech. degree in Computer Science and engineering at University of Calicut, and doing MTech in Computer Science at KMCT College of Engineering, Calicut. She was a Performance Testing Engineer from 2006 to 2014. Her research interest include Image Processing and Machine Learning 